\title{
Interpretation of the $\mathrm{LHCb} \boldsymbol{P}_{c}$ States as Hadronic Molecules and Hints of a Narrow $P_{c}(4380)$
}

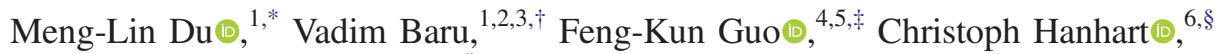 \\ Ulf-G. Meißner $\oplus^{1,6,7, \|}$ José A. Oller $\oplus^{8, \uparrow}$ and Qian Wang $\oplus^{9,10, * *}$ \\ ${ }^{1}$ Helmholtz-Institut für Strahlen- und Kernphysik and Bethe Center for Theoretical Physics, Universität Bonn, \\ D-53115 Bonn, Germany \\ ${ }^{2}$ Institute for Theoretical and Experimental Physics NRC "Kurchatov Institute", Moscow 117218, Russia \\ ${ }^{3}$ P.N. Lebedev Physical Institute of the Russian Academy of Sciences, Leninskiy Prospect 53, Moscow 119991, Russia \\ ${ }^{4}$ CAS Key Laboratory of Theoretical Physics, Institute of Theoretical Physics, Chinese Academy of Sciences, \\ Zhong Guan Cun East Street 55, Beijing 100190, China \\ ${ }^{5}$ School of Physical Sciences, University of Chinese Academy of Sciences, Beijing 100049, China \\ ${ }^{6}$ Institute for Advanced Simulation, Institut für Kernphysik and Jülich Center for Hadron Physics, \\ Forschungszentrum Jülich, D-52425 Jülich, Germany \\ ${ }^{7}$ Tbilisi State University, 0186 Tbilisi, Georgia \\ ${ }^{8}$ Departamento de Física, Universidad de Murcia, E-30071 Murcia, Spain \\ ${ }^{9}$ Guangdong Provincial Key Laboratory of Nuclear Science, Institute of Quantum Matter, South China Normal University, \\ Guangzhou 510006, China \\ ${ }^{10}$ Theoretical Physics Center for Science Facilities, Institute of High Energy Physics, Chinese Academy of Sciences, \\ Beijing 100049, China
}

(Received 5 November 2019; revised manuscript received 13 January 2020; accepted 24 January 2020; published 19 February 2020)

\begin{abstract}
Three hidden-charm pentaquark $P_{c}$ states, $P_{c}(4312), P_{c}(4440)$, and $P_{c}(4457)$ were revealed in the $\Lambda_{b}^{0} \rightarrow J / \psi p K^{-}$process measured by LHCb using both run I and run II data. Their nature is under lively discussion, and their quantum numbers have not been determined. We analyze the $J / \psi p$ invariant mass distributions under the assumption that the crossed-channel effects provide a smooth background. For the first time, such an analysis is performed employing a coupled-channel formalism with the scattering potential involving both one-pion exchange as well as short-range operators constrained by heavy quark spin symmetry. We find that the data can be well described in the hadronic molecular picture, which predicts seven $\Sigma_{c}^{(*)} \bar{D}^{(*)}$ molecular states in two spin multiplets, such that the $P_{c}(4312)$ is mainly a $\Sigma_{c} \bar{D}$ bound state with $J^{P}=1 / 2^{-}$, while $P_{c}(4440)$ and $P_{c}(4457)$ are $\Sigma_{c} \bar{D}^{*}$ bound states with quantum numbers $3 / 2^{-}$and $1 / 2^{-}$, respectively. We also show that there is evidence for a narrow $\Sigma_{c}^{*} \bar{D}$ bound state in the data which we call $P_{c}(4380)$, different from the broad one reported by LHCb in 2015 . With this state included, all predicted $\Sigma_{c} \bar{D}, \Sigma_{c}^{*} \bar{D}$, and $\Sigma_{c} \bar{D}^{*}$ hadronic molecules are seen in the data, while the missing three $\Sigma_{c}^{*} \bar{D}^{*}$ states are expected to be found in future runs of the LHC or in photoproduction experiments.
\end{abstract}

DOI: 10.1103/PhysRevLett.124.072001

Introduction.-The confinement property of quantum chromodynamics (QCD) in principle allows for the existence of a large variety of color neutral objects. However, it is not clear yet which configurations are realized in nature. As a result, searching for multiquark exotic hadrons beyond the conventional quark model has been one of the central issues in the study of the strong interactions. Tremendous

Published by the American Physical Society under the terms of the Creative Commons Attribution 4.0 International license. Further distribution of this work must maintain attribution to the author(s) and the published article's title, journal citation, and DOI. Funded by SCOAP . developments have been made in the new era since 2003 when the $B$ factories discovered the $D_{s 0}^{*}(2317)$ [1] and $X(3872)$ [2], whose properties are in notable contradiction with quark model predictions. The interest in studying such exotic hadrons was further boosted by the LHCb discovery of the hidden-charm pentaquarks $P_{c}(4450)$ and $P_{c}(4380)$ decaying into $J / \psi p$ in the $\Lambda_{b}^{0} \rightarrow K^{-} J / \psi p$ process in 2015 [3]. The experimental and theoretical efforts are summarized in a number of comprehensive reviews [4-16]. When $\mathrm{LHCb}$ updated their measurements with a one-order-ofmagnitude larger data sample [17], the narrow $P_{c}(4450)$ appeared to be split into two narrower structures $P_{c}(4440)$ and $P_{c}(4457)$, and a third narrow peak $P_{c}(4312)$ showed 
up. At the same time, the broad $P_{c}(4380)$ (whose existence needs to be verified in a complete amplitude analysis that is under way) lost its significance. A cornucopia of theoretical interpretations followed these new discoveries, including models of hadronic molecules [18-39], compact pentaquark states [40-45], and hadrocharmonia [46]. An amplitude analysis was performed in Ref. [47] focusing on the $P_{c}(4312)$ which was suggested to be a virtual state. Among the explanations, the hadronic molecular model stands out as it explains all of the three narrow $P_{c}$ states simultaneously as $\Sigma_{c} \bar{D}$ [for $\left.P_{c}(4312)\right]$ and $\Sigma_{c} \bar{D}^{*}$ [for $P_{c}(4440)$ and $P_{c}(4457)$ ] bound states, see, e.g., Refs. [21,26,32], employing the approximate heavy quark spin symmetry (HQSS) of QCD. However, the model predicts in addition four more states, including one $\Sigma_{c}^{*} \bar{D}$ state at around 4.37 to $4.38 \mathrm{GeV}$ and three states slightly below the $\Sigma_{c}^{*} \bar{D}^{*}$ threshold. These seven $P_{c}$ states are contained in two heavy quark spin multiplets, labeled as $j_{\ell}^{P}$, with $j_{\ell}$ and $P$ the total angular momentum of the light degrees of freedom and parity, respectively: three with $j_{\ell}^{P}=\frac{1}{2}^{-}$and four with $j_{\ell}^{P}=\frac{3}{2}$. States in these two multiplets with the same spin mix because the $\Sigma_{c}(\bar{D})$ is not degenerate with the $\Sigma_{c}^{*}\left(\bar{D}^{*}\right)$. While only three of the states correspond to the ones reported by $\mathrm{LHCb}$, it is crucial to check whether the existence of the whole two multiplets is consistent with the $J / \psi p$ distribution measured by LHCb. This is the question addressed in this Letter: by constructing coupledchannel amplitudes analogous to those used in the analysis of the $Z_{b}$ states [48-51], we show that the observed $J / \psi p$ invariant mass distribution can be well described in the hadronic molecular scenario with seven $\Sigma_{c}^{(*)} \bar{D}^{(*)}$ molecules, where the $P_{c}(4312)$ and $P_{c}(4440) / P_{c}(4457)$ are $\Sigma_{c} \bar{D}$ and $\Sigma_{c} \bar{D}^{*}$ bound states, respectively. For the first time, we point out a clear signal from data for the existence of a narrow $P_{c}(4380)$ as a $\Sigma_{c}^{*} \bar{D}$ bound state. The three predicted $\Sigma_{c}^{*} \bar{D}^{*}$ states still await discovery. In addition, we emphasize that the inclusion of the one-pion exchange (OPE) allows one to single out a unique solution corresponding to the global minimum instead of two equivalent solutions present in the pure contact case.

Framework.-In order to describe the measured $J / \psi p$ distribution, we construct coupled-channel amplitudes considering all the $\Sigma_{c}^{(*)} \bar{D}^{(*)}$ channels (called elastic channels $[48,49]$, since their thresholds are close to the $P_{c}$ masses) and the $J / \psi p$ channels (inelastic channels). HQSS is used to relate all the $\Sigma_{c}^{(*)} \bar{D}^{(*)}$ channels [21,32,52,53], and their couplings to the $J / \psi p[31,32]$. We also allow for additional inelastic channels not included explicitly in the amplitudes. To this end, we expand the two-particle states in the basis of HQSS eigenstates $\left|s_{Q} \otimes j_{\ell}\right\rangle$, with $s_{Q}$ and $j_{\ell}$ representing the total spin of the heavy quarks and total angular momentum of light degrees of freedom, respectively. In this notation, the $\Sigma^{(*)}$ and $\bar{D}^{(*)}$ spin multiplets are $\left|\frac{1}{2} \otimes 1\right\rangle$ and $\left|\frac{1}{2} \otimes \frac{1}{2}\right\rangle$, respectively. One can rewrite the $S$-wave $\Sigma_{c}^{(*)} \bar{D}^{(*)}$ systems in terms of $\left|s_{Q} \otimes j_{\ell}\right\rangle$ as [32,52]

$$
\begin{gathered}
\left(\begin{array}{c}
\left|\Sigma_{c} \bar{D}\right\rangle \\
\left|\Sigma_{c} \bar{D}^{*}\right\rangle \\
\left|\Sigma_{c}^{*} \bar{D}^{*}\right\rangle
\end{array}\right)_{\frac{1}{2}}=\left(\begin{array}{ccc}
\frac{1}{2} & \frac{-1}{2 \sqrt{3}} & \sqrt{\frac{2}{3}} \\
\frac{-1}{2 \sqrt{3}} & \frac{5}{6} & \frac{\sqrt{2}}{3} \\
\sqrt{\frac{2}{3}} & \frac{\sqrt{2}}{3} & -\frac{1}{3}
\end{array}\right)\left(\begin{array}{l}
\left|0 \otimes \frac{1}{2}\right\rangle \\
\left|1 \otimes \frac{1}{2}\right\rangle \\
\left|1 \otimes \frac{3}{2}\right\rangle
\end{array}\right), \\
\left(\begin{array}{c}
\left|\Sigma_{c} \bar{D}^{*}\right\rangle \\
\left|\Sigma_{c}^{*} \bar{D}\right\rangle \\
\left|\Sigma_{c}^{*} \bar{D}^{*}\right\rangle
\end{array}\right)_{\frac{3}{2}}=\left(\begin{array}{ccc}
\frac{-1}{\sqrt{3}} & \frac{1}{3} & \frac{\sqrt{5}}{3} \\
\frac{1}{2} & \frac{-1}{\sqrt{3}} & \frac{1}{2} \sqrt{\frac{5}{3}} \\
\frac{1}{2} \sqrt{\frac{5}{3}} & \frac{\sqrt{5}}{3} & \frac{1}{6}
\end{array}\right)\left(\begin{array}{l}
\left|0 \otimes \frac{3}{2}\right\rangle \\
\left|1 \otimes \frac{1}{2}\right\rangle \\
\left|1 \otimes \frac{3}{2}\right\rangle
\end{array}\right), \\
\left|\Sigma_{c}^{*} \bar{D}^{*}\right\rangle_{5 / 2}=\left|1 \otimes \frac{3}{2}\right\rangle
\end{gathered}
$$

where the subscripts on the left-hand side represent the total angular momentum $J=\frac{1}{2}, \frac{3}{2}$, and $\frac{5}{2}$. The rotation matrices in Eqs. (1)-(3) will be denoted as $R^{J}$ in the following. One can obtain the contact terms for $S$-wave interactions of the elastic channels in terms of two independent matrix elements,

$$
\begin{aligned}
& C_{1 / 2} \equiv\left\langle s_{Q} \otimes \frac{1}{2}\left|\hat{\mathcal{H}}_{I}\right| s_{Q} \otimes \frac{1}{2}\right\rangle, \\
& C_{3 / 2} \equiv\left\langle s_{Q} \otimes \frac{3}{2}\left|\hat{\mathcal{H}}_{I}\right| s_{Q} \otimes \frac{3}{2}\right\rangle,
\end{aligned}
$$

with $\hat{\mathcal{H}}_{I}$ the effective Hamiltonian respecting HQSS. In the heavy quark limit, the contact interactions defined above are independent of $s_{Q}=0,1$. Since we work to leading order, the above matrix elements are constants. In particular, the $D$-wave $\Sigma_{c}^{(*)} \bar{D}^{(*)}$ contact terms, which turned out to be necessary in the study of the $Z_{b}$ states [51], will be neglected since data in the inelastic channels are insensitive to such operators. The contact terms in the particle basis are

$$
C_{\alpha \beta}^{J}=\sum_{n_{J}} R_{\alpha n_{J}}^{J} C_{j_{\ell}\left(n_{J}\right)}\left(R^{J}\right)_{n_{J} \beta}^{T},
$$

where $j_{\ell}\left(n_{J}\right)$ denotes the light-quark spin of the $n$th channel for a given $J$ multiplet.

The OPE potential can be obtained using the effective Lagrangian for the axial coupling of the pions to the charmed mesons and baryons [54,55]

$$
\mathcal{L}=\frac{g}{4}\left\langle\boldsymbol{\sigma} \cdot \boldsymbol{u}_{a b} \bar{H}_{b} \bar{H}_{a}^{\dagger}\right\rangle-i g_{1} \epsilon^{i j k} \operatorname{Tr}\left[S_{i}^{\dagger} u_{j} S_{k}\right],
$$

where $\langle$.$\rangle and \operatorname{Tr}[$.$] denote traces in the spinor and isospin$ spaces, respectively, $\sigma$ represents the Pauli matrices, $S_{i}$ and $\bar{H}$ are the heavy quark spin doublets for ground states $\left(\Sigma_{c}, \Sigma_{c}^{*}\right)$ and $\left(\bar{D}, \bar{D}^{*}\right)[56]$, 


$$
\boldsymbol{S}=\frac{1}{\sqrt{3}} \boldsymbol{\sigma} \Sigma_{c}+\boldsymbol{\Sigma}_{c}^{*}, \quad \bar{H}=-\bar{D}+\boldsymbol{\sigma} \cdot \overline{\boldsymbol{D}}^{*},
$$

$\boldsymbol{u}=-i \nabla \Phi / F_{\pi}+\mathcal{O}\left(\Phi^{3}\right), \Phi=\boldsymbol{\tau} \cdot \boldsymbol{\pi}$ with $\boldsymbol{\tau}$ and $\boldsymbol{\pi}$ the Pauli matrices in the isospin space and the pion fields, in order, and $F_{\pi}=92.1 \mathrm{MeV}$ is the pion decay constant. From the measured width of $D^{*+} \rightarrow D^{0} \pi^{+}$one gets $g=0.57$ [57], and the coupling $g_{1}=0.42$ is taken from the lattice QCD calculation [58]. The OPE contributes to both $S$ and $D$ waves and can be important for describing the line shapes around thresholds [50,51,59].

Also the transitions between the elastic and inelastic channels can be related via HQSS. While the $\left|1 \otimes \frac{1}{2}\right\rangle$ component in Eqs. (1)-(3) couples to $J / \psi p$ in the $S$ wave in the heavy quark limit, the $\left|1 \otimes \frac{3}{2}\right\rangle$ only couples to $J / \psi p$ in the $D$ wave. We introduce two coupling strengths,

$g_{S} \equiv\left\langle 1 \otimes \frac{1}{2}\left|\hat{\mathcal{H}}_{I}\right| J / \psi p\right\rangle_{S}, \quad g_{D} k^{2} \equiv\left\langle 1 \otimes \frac{3}{2}\left|\hat{\mathcal{H}}_{I}\right| J / \psi p\right\rangle_{D}$,

where $k$ is the magnitude of the $J / \psi$ three-momentum in the c.m. frame of $J / \psi p$. Then, the transition vertices $\mathcal{V}_{\alpha i}^{J}$ between the $\alpha$ th elastic and $i$ th inelastic channel, with $i=1,2$ denoting the $S$-wave and $D$-wave $J / \psi p$, respectively, can be easily obtained by virtue of the decompositions in Eqs. (1)-(3) as

$$
\begin{aligned}
& \mathcal{V}_{\alpha 1}^{J}=g_{S} R_{\alpha 2}^{J}, \quad \mathcal{V}_{\alpha 2}^{J}(k)=g_{D} k^{2} R_{\alpha 3}^{J}, \quad J=\frac{1}{2}, \frac{3}{2}, \\
& \mathcal{V}_{\alpha 1}^{J}=0, \quad \mathcal{V}_{\alpha 2}^{J}(k)=g_{D} k^{2}, \quad J=\frac{5}{2} .
\end{aligned}
$$

The direct $J / \psi p$ interaction is Okubo-Zweig-Iizuka suppressed and found very weak in a recent lattice QCD study [60]. Thus, the inelastic $J / \psi p$ channel is only included through its coupling to the elastic channels [48-51,61]. While the real part of its contribution can be absorbed by redefining the contact terms $C_{\alpha \beta}^{J}$ [51], the imaginary part cannot. We thus introduce

$$
V_{J / \psi p, \alpha \beta}^{J}(E)=-\frac{i}{2 \pi E} \sum_{j=1}^{2} m_{J / \psi} m_{p} \mathcal{V}_{\alpha j}^{J} \mathcal{V}_{\beta j}^{J} k
$$

into the effective elastic potential. It is expected that, in addition to the $J / \psi p$ channels, there are more inelastic channels, most prominently $\Lambda_{c} \bar{D}^{(*)}$ and $\eta_{c} p[31,62,63]$. While the latter is connected to the $J / \psi p$ channels via HQSS, the former is not and thus we are obliged to parametrize especially those via an additional imaginary part of the two contact terms. This introduces two more parameters. Thus the scattering problem contains in total 6 parameters and the full effective potential for the elastic channels can be written as

$$
V^{J}(E, p, q)=C^{J}+V_{J / \psi p}^{J}(E)+V_{\mathrm{OPE}}^{J}(E, p, q),
$$

with $q$ and $p$ for the incoming and outgoing relative momenta of the corresponding channels, respectively, and $E$ for the total energy. The explicit expressions for the matrix $V_{\mathrm{OPE}}^{J}(E, p, q)$ are provided in Ref. [64].

For the weak production amplitude for $\Lambda_{b} \rightarrow K^{-} \Sigma_{c}^{(*)} \bar{D}^{(*)}$ we only consider the elastic $\Sigma_{c}^{(*)} \bar{D}^{(*)}$ channels produced in an $S$ wave, since the energy region of interest is close to the $\Sigma_{c}^{(*)} \bar{D}^{(*)}$ thresholds. Then the weak production matrix elements may be expressed as $\mathcal{F}_{n}^{J}=\left\langle\Lambda_{b}\left|\hat{\mathcal{H}}_{W}\right| K^{-}\right.$ $\left.\left(s_{Q} \otimes j_{\ell}\right)_{n}^{J}\right\rangle$, where $\left(s_{Q} \otimes j_{\ell}\right)_{n}^{J}$ refers to the $n$th state in the $\left|s_{Q} \otimes j_{\ell}\right\rangle$ basis in Eqs. (1)-(3). The production contact term for the $\alpha$ th elastic channel for a given $J$ then reads

$$
P_{\alpha}^{J}=\sum_{n} R_{\alpha n}^{J} \mathcal{F}_{n}^{J}
$$

In total, there are seven additional parameters $\mathcal{F}_{n}^{J}$.

With the above ingredients, one can obtain the production amplitude, $U_{\alpha}^{J}$, for the $\alpha^{\text {th }}$ elastic channel by solving the following Lippmann-Schwinger equations (LSEs),

$$
\begin{aligned}
& U_{\alpha}^{J}(E, p) \\
& \quad=P_{\alpha}^{J}-\sum_{\beta} \int \frac{d^{3} \boldsymbol{q}}{(2 \pi)^{3}} V_{\alpha \beta}^{J}(E, p, q) G_{\beta}(E, q) U_{\beta}^{J}(E, q),
\end{aligned}
$$

and for the $i$ th $J / \psi p$ inelastic channel $U_{i}^{J}$ via

$U_{i}^{J}(E, k)=-\sum_{\beta} \int \frac{d^{3} \boldsymbol{q}}{(2 \pi)^{3}} \mathcal{V}_{i \beta}^{J}(k) G_{\beta}(E, q) U_{\beta}^{J}(E, q)$.

The two-body propagator is

$G_{\beta}(E, q)=\frac{2 \mu_{\beta}}{q^{2}-p_{\beta}^{2}-i \epsilon}, \quad p_{\beta}^{2} \equiv 2 \mu_{\beta}\left(E-m_{\mathrm{th}}^{\beta}\right)$,

with $\mu_{\beta}$ and $m_{\mathrm{th}}^{\beta}$ the reduced mass and the threshold of the $\beta$ th elastic channel. The $\Sigma_{c}^{(*)}$ widths of $1.86 \mathrm{MeV}(15 \mathrm{MeV})$ [57] are accounted for using a complex mass $m-i \Gamma / 2$ in $m_{\mathrm{th}}^{\beta}$. The LSE is regularized using a hard cutoff, varied in the range from 1 to $1.5 \mathrm{GeV}$. Since the results barely depend on its value (effects of the cutoff variation can be largely absorbed into the refitted contact terms), the final results will be presented for the cutoff of $1 \mathrm{GeV}$. The equations given are unitary as long as the additional imaginary parts of the contact terms are omitted. Unitarity can be restored once data on the $\Lambda_{c} \bar{D}^{(*)}$ channels are available, and we checked that introducing the $\Lambda_{c} \bar{D}^{*}$ channel in the way of Eq. (8) did not produce a sizable difference. 
In order to fit the $J / \psi p$ invariant mass distribution, an incoherent smooth background is used to model possible contributions from misidentified non- $\Lambda_{b}^{0}$ events, the $\Lambda^{*}$ resonances coupled to $p K^{-}$, and possibly additional broad $P_{c}^{+}$structures. Here we use the form

$f_{\text {bgd }}(E)=b_{0}+b_{1} E^{2}+b_{2} E^{4}+\left|\frac{g_{r}}{m^{2}-E^{2}-i \Gamma E}\right|^{2}$,

which contains the parameters $b_{0}, b_{1}, b_{2}, g_{r}, m$, and $\Gamma$. The backgrounds used in the experimental analysis [17] are also considered, and the results are similar, which will be included in the uncertainties. We perform fits employing the potential of Eq. (9) either omitting (scheme I) or including (scheme II) $V_{\text {OPE }}^{J}$.

Results and discussions.-In this analysis, we do not consider isospin symmetry breaking effects which can be important to give rise to isospin-breaking decay modes $[20,65]$ but have little effect on the description of the line shapes in the isospin-conserving $J / \psi p$ channel. Convolution with the experimental energy resolution is considered. For scheme I we find two solutions, denoted as $A$ and $B$, describing the data almost equally well (with $\chi^{2} /$ d.o.f. $=1.01$ and 1.03 , respectively). The corresponding best fits are shown in the left panel of Fig. 1. The two solutions produce different values of the parameters, in particular $C_{1 / 2}$ and $C_{3 / 2}$ (in fact, the values of $C_{1 / 2}$ and $C_{3 / 2}$ in solution $A$ are very close to those of $C_{3 / 2}$ and $C_{1 / 2}$ in solution $B$, respectively), and thus give different pole locations. However, both solutions give seven poles in the $\Sigma_{c}^{(*)} \bar{D}^{(*)}$ scattering amplitudes, i.e., seven $P_{c}$ states: three with $J=1 / 2$, three with $J=3 / 2$ and one with $J=5 / 2$. The masses of the generated $P_{c}$ states in solutions $A$ and $B$ are close to those of scenarios $A$ and $B$ for the

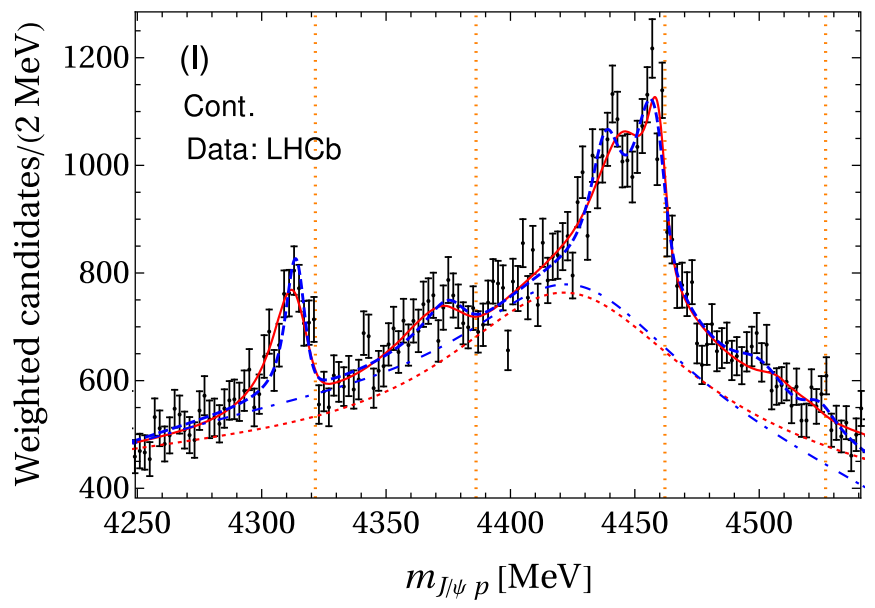

corresponding quantum numbers in Refs. [21,32], respectively, though the widths are larger due to the inelastic channels and the $\Sigma_{c}^{(*)}$ widths, and thus are not shown here.

In both solutions, among the seven poles, the lowest one corresponds to the $P_{c}(4312)$ with $\frac{1}{2}^{-}$, and is a $\Sigma_{c} \bar{D}$ bound state: it is located in the second Riemann sheet (of the $J / \psi p$ channel), and would become a real bound state pole in the first Riemann sheet if the $J / \psi p$ channel were switched off. This is different from the virtual state scenario of $\Sigma_{c} \bar{D}$ in Ref. [47] which only fits to data around the $\Sigma_{c} \bar{D}$ threshold.

There are two $\Sigma_{c} \bar{D}^{*}$ bound states with quantum numbers $\frac{1}{2}-$ and $\frac{3-}{2}$, corresponding to the $P_{c}(4440)$ and the $P_{c}(4457)$, respectively, in solution $A$ and interchanged in solution $B$. The mass pattern of the three $P_{c}$ states dominated by the $\Sigma_{c}^{*} \bar{D}^{*}$ channel is analogous to that of the $\Sigma_{c} \bar{D}^{*}$ channel, i.e., $m_{1 / 2^{-}}<m_{3 / 2^{-}}<m_{5 / 2^{-}}$for solution $A$ and the opposite for solution $B$. In both solutions, there is a narrow pole around $4.38 \mathrm{GeV}$ whose dominant component is $\Sigma_{c}^{*} \bar{D}$ (see also Ref. [26]). This means that HQSS requires the existence of a $P_{c}(4380)$ resonance which, however, is narrow and thus different from the broad resonance reported by $\mathrm{LHCb}$ in 2015 [3].

In scheme II the OPE is included. The importance of its tensor force is well known for the nucleon-nucleon interaction. It leads to the mixing between $S$ and $D$ waves and can have a sizable impact on the line shape between thresholds $[50,51,59]$. Unlike in scheme I, once the full OPE is included, there is only one solution corresponding to the best fit with $\chi^{2} /$ d.o.f. $=0.98$, shown in the right panel of Fig. 1. It leads to poles presented in Table I which are similar to those in solution $B$ of scheme I (see also Refs. $[35,66])$. The pole positions, the dominant channels (DCs) having the largest effective couplings (derived form residues of the $T$ matrix), and effective couplings of these

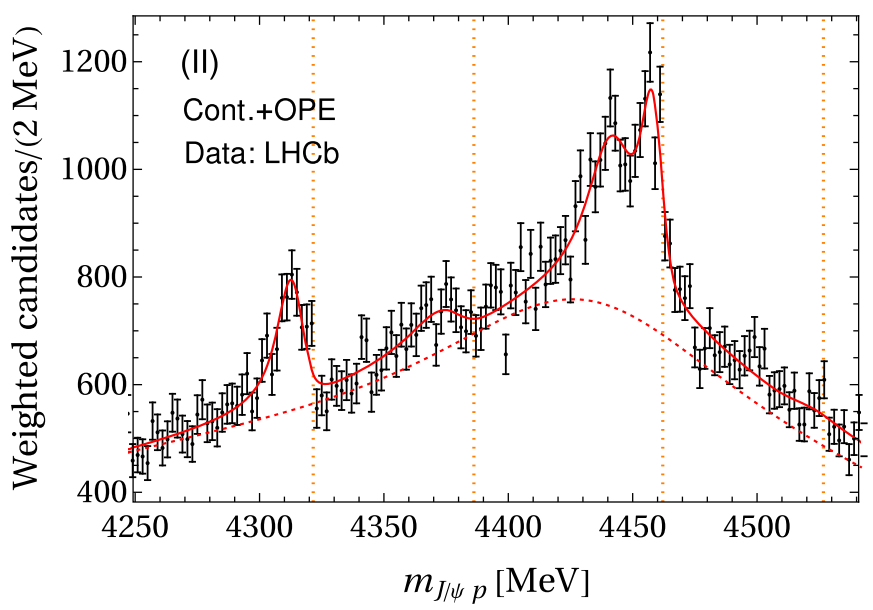

FIG. 1. Left panel: the fitted invariant mass distributions vs the weighted experimental data [17] for both solution $A$ (blue dashed curves) and solution $B$ (red solid ones) of scheme I. The corresponding backgrounds are shown as blue dash-dotted and red dotted curves, respectively. Right panel: the best fit for scheme II with the dotted curve representing the background. The vertical dashed lines in both panels from left to right are the $\Sigma_{c} \bar{D}, \Sigma_{c}^{*} \bar{D}, \Sigma_{c} \bar{D}^{*}$, and $\Sigma_{c}^{*} \bar{D}^{*}$ thresholds, respectively. 
TABLE I. The names of the states, their quantum numbers found from the fits within scheme II, the pole positions (on the sheets close to the physical one), the dominant channels (DCs) and their thresholds, the dimensionless couplings of the resonances in the DCs (from the $T$-matrix residues and defined as $G_{\mathrm{DC}}$ ), and the resonance couplings to the source derived from the residues, which are normalized by the event numbers and thus only the relative values are meaningful. The uncertainties given are from taking different backgrounds, the uncertainties from the fit for a given background are negligible. The $P_{c}(4380)$ in boldface is the new state we advocate in this work.

\begin{tabular}{llcccc}
\hline \hline Scheme II & $J^{P}$ & Pole $[\mathrm{MeV}]$ & DC $($ threshold $[\mathrm{MeV}])$ & $G_{\mathrm{DC}}$ & Production \\
\hline$P_{c}(4312)$ & $\frac{1}{2}-$ & $4314(2)-5(2) i$ & $\Sigma_{c} \bar{D}(4321.6)$ & $2.86(12)-0.44(24) i$ & $636(73)-98(53) i$ \\
$\mathbf{P}_{\mathbf{c}}(\mathbf{4 3 8 0})$ & $\frac{3}{2}$ & $4378(2)-13(3) i$ & $\Sigma_{c}^{*} \bar{D}(4386.2)$ & $3.00(12)-0.49(27) i$ & $618(373)-181(95) i$ \\
$P_{c}(4440)$ & $\frac{3}{2}-$ & $4441(2)-11(3) i$ & $\Sigma_{c} \bar{D}^{*}(4462.1)$ & $3.91(11)-0.62(19) i$ & $999(140)-15(18) i$ \\
$P_{c}(4457)$ & $\frac{1}{2}-$ & $4459(2)-4(1) i$ & $\Sigma_{c} \bar{D}^{*}(4462.1)$ & $2.09(17)-0.46(18) i$ & $-918(68)+159(78) i$ \\
$P_{c}$ & $\frac{1}{2}-$ & $4524(2)-9(1) i$ & $\Sigma_{c}^{*} \bar{D}^{*}(4526.7)$ & $1.90(23)-0.28(21) i$ & $-228(384)+22(23) i$ \\
$P_{c}$ & $\frac{3}{2}$ & $4518(2)-11(2) i$ & $\Sigma_{c}^{*} \bar{D}^{*}(4526.7)$ & $2.83(16)-0.43(18) i$ & $-156(517)-58(43) i$ \\
$P_{c}$ & $\frac{5}{2}-$ & $4498(5)-35(17) i$ & $\Sigma_{c}^{*} \bar{D}^{*}(4526.7)$ & $4.66(55)-1.12(32) i$ & $-393(620)-2(26) i$ \\
\hline \hline
\end{tabular}

are listed in Table I. The results are insensitive to the form of the background, and the effects of using different backgrounds give the errors in Table I (the statistical errors propagating from the data are much smaller).

One sees that the $P_{c}(4312)$ couples dominantly to $\Sigma_{c} \bar{D}$ with $J^{P}=\frac{1}{2}^{-}$, and both the $P_{c}(4440)$ and $P_{c}$ (4457) couple dominantly to the $\Sigma_{c} \bar{D}^{*}$ with quantum numbers $\frac{3}{2}^{-}$and $\frac{1}{2}^{-}$, respectively. They are all bound state poles and should be understood as hadronic molecules of the corresponding channels [11]. Comparing the fits from scheme I and scheme II, one sees that the OPE helps making the dip between the $P_{c}(4440)$ and $P_{c}(4457)$ more evident. In both schemes there is a narrow $P_{c}(4380)$ located at the right position where the data show a peak, though less prominent than those of the well-known three $P_{c}$ states. Its existence and properties are a consequence of HQSS in the hadronic molecular picture. We have checked that it persists no matter whether or not the data around $4.38 \mathrm{GeV}$ are included in the fit. The absolute value of the production strength of the $P_{c}(4380)$ shown in the last column of the table differs from zero by $1.7 \sigma$ which might be taken as a measure of the significance of this resonance in the data. The three $\Sigma_{c}^{*} \bar{D}^{*}$ molecules, which are expected to exist $[21,32,37,52,53]$, do not have any unambiguous signal in the data. Their production strengths related to the residues of the production amplitude $U_{\alpha}^{J}$ at the pole suggest that they are less strongly produced in $\Lambda_{b}$ decays. One possible reason could be that the production of the three most pronounced $P_{c}$ structures gets enhanced by nearby triangle singularities discussed in Refs. [17,67-69].

Summary and outlook.-In summary, we investigated for the first time whether the appealing hadronic molecular model for the observed $P_{c}$ states is consistent with the $\mathrm{LHCb}$ data. A coupled-channel formalism is used to analyze the $J / \psi p$ invariant mass distribution, which contains much more information than the extracted pentaquark masses only. The relevant effective potential constructed based on HQSS involves all transitions between the elastic
$\Sigma_{c}^{(*)} \bar{D}^{(*)}$ channels, transitions from the elastic to the $S$ - and $D$-wave $J / \psi p$ inelastic channels as well as the coupling to additional effective inelastic channels. We find that the data can be well described. In addition to the three established states, in our analysis a narrow $P_{c}(4380)$ state, identified as a $\frac{3}{2}-\Sigma_{c}^{*} \bar{D}$ molecule, with its properties fixed by HQSS, shows up as a clear signal in the data. The three $\Sigma_{c}^{*} \bar{D}^{*}$ bound states with masses from around 4.49 to $4.52 \mathrm{GeV}$ are almost invisible because of their relatively low production rates in the $\Lambda_{b}$ decays. We expect that they can be resolved in the forthcoming data to be collected at the LHC run3 period or other production processes, such as the $J / \psi$ photoproduction [63,70-78]. It should be stressed that, if the $P_{c}$ states indeed are hadronic molecules, they have to show up as prominent structures also in the elastic channels, and data for those would therefore be extremely valuable. To further refine our approach, data are needed in the $\Lambda_{c} \bar{D}^{(*)}$ as well as in the $\eta_{c} p$ channels. The latter would provide additional information on the amount of spin symmetry violation in the system. All these studies will shed important light on our understanding of how QCD forms hadrons.

We are grateful to Marek Karliner, Misha Mikhasenko, Sebastian Neubert, Juan Nieves, and Qiang Zhao for useful comments and discussions. This work is supported in part by the National Natural Science Foundation of China (NSFC) and the Deutsche Forschungsgemeinschaft (DFG) through the funds provided to the Sino-German Collaborative Research Center "Symmetries and the Emergence of Structure in QCD" (NSFC Grant No. 11621131001 and DFG Grant No. TRR110), by the NSFC under Grants No. 11835015, No. 11947302, and No. 11961141012, by the Chinese Academy of Sciences (CAS) under Grants No. QYZDB-SSW-SYS013 and No. XDPB09, by the CAS Center for Excellence in Particle Physics (CCEPP), and by the Munich Institute for Astro- and Particle Physics (MIAPP) of the DFG cluster 
of excellence "Origin and Structure of the Universe." Q. W. is also supported by the Thousand Talents Plan for Young Professionals and research startup funding at SCNU. The work of U. G. M. is further supported by the CAS President's International Fellowship Initiative (PIFI) (Grant No. 2018DM0034) and by the VolkswagenStiftung (Grant No. 93562). The work of V. B. is supported by the Russian Science Foundation (Grant No. 18-12-00226). J. A. O. would like to acknowledge partial financial support by the MINECO (Spain) and FEDER Grant No. FPA201677313-P.

*du@hiskp.uni-bonn.de

${ }^{\dagger}$ vadim.baru@tp2.rub.de

*fkguo@itp.ac.cn

§c.hanhart@fz-juelich.de

"meissner@hiskp.uni-bonn.de

qoller@um.es

**aianwang@m.scnu.edu.cn

[1] B. Aubert et al. (BABAR Collaboration), Phys. Rev. Lett. 90, 242001 (2003).

[2] S. K. Choi et al. (Belle Collaboration), Phys. Rev. Lett. 91, 262001 (2003).

[3] R. Aaij et al. (LHCb Collaboration), Phys. Rev. Lett. 115, 072001 (2015).

[4] N. Brambilla et al., Eur. Phys. J. C 71, 1534 (2011).

[5] H. X. Chen, W. Chen, X. Liu, Y. R. Liu, and S. L. Zhu, Rep. Prog. Phys. 80, 076201 (2017).

[6] H. X. Chen, W. Chen, X. Liu, and S. L. Zhu, Phys. Rep. 639, 1 (2016)

[7] Y. Dong, A. Faessler, and V. E. Lyubovitskij, Prog. Part. Nucl. Phys. 94, 282 (2017).

[8] A. Esposito, A. Pilloni, and A. D. Polosa, Phys. Rep. 668, 1 (2017).

[9] A. Hosaka, T. Iijima, K. Miyabayashi, Y. Sakai, and S. Yasui, Prog. Theor. Exp. Phys. 2016, 062 C01 (2016).

[10] S. L. Olsen, T. Skwarnicki, and D. Zieminska, Rev. Mod. Phys. 90, 015003 (2018).

[11] F.-K. Guo, C. Hanhart, U.-G. Meißner, Q. Wang, Q. Zhao, and B. S. Zou, Rev. Mod. Phys. 90, 015004 (2018).

[12] E. Kou et al. (Belle-II Collaboration), Prog. Theor. Exp. Phys. 2019, 123C01 (2019).

[13] A. Cerri et al., CERN Yellow Rep. Monogr. 7, 867 (2019).

[14] Y. R. Liu, H. X. Chen, W. Chen, X. Liu, and S. L. Zhu, Prog. Part. Nucl. Phys. 107, 237 (2019).

[15] N. Brambilla, S. Eidelman, C. Hanhart, A. Nefediev, C. P. Shen, C. E. Thomas, A. Vairo, and C. Z. Yuan, arXiv:1907 .07583

[16] F.-K. Guo, X.-H. Liu, and S. Sakai, arXiv:1912.07030.

[17] R. Aaij et al. (LHCb Collaboration), Phys. Rev. Lett. 122, 222001 (2019).

[18] H. X. Chen, W. Chen, and S. L. Zhu, Phys. Rev. D 100, 051501(R) (2019).

[19] R. Chen, Z. F. Sun, X. Liu, and S. L. Zhu, Phys. Rev. D 100, 011502(R) (2019).

[20] F.-K. Guo, H.-J. Jing, U.-G. Meißner, and S. Sakai, Phys. Rev. D 99, 091501(R) (2019).
[21] M. Z. Liu, Y. W. Pan, F. Z. Peng, M. S. Sánchez, L. S. Geng, A. Hosaka, and M. P. Valderrama, Phys. Rev. Lett. 122, 242001 (2019).

[22] J. He, Eur. Phys. J. C 79, 393 (2019).

[23] Z. H. Guo and J. A. Oller, Phys. Lett. B 793, 144 (2019).

[24] Y. Shimizu, Y. Yamaguchi, and M. Harada, arXiv:1904 .00587.

[25] C. J. Xiao, Y. Huang, Y. B. Dong, L. S. Geng, and D. Y. Chen, Phys. Rev. D 100, 014022 (2019).

[26] C. W. Xiao, J. Nieves, and E. Oset, Phys. Rev. D 100, 014021 (2019).

[27] F. L. Wang, R. Chen, Z. W. Liu, and X. Liu, arXiv:1905 .03636 .

[28] L. Meng, B. Wang, G. J. Wang, and S. L. Zhu, Phys. Rev. D 100, 014031 (2019).

[29] J. J. Wu, T.-S. H. Lee, and B. S. Zou, Phys. Rev. C 100, 035206 (2019)

[30] C.-W. Xiao, J. Nieves, and E. Oset, Phys. Lett. B 799, 135051 (2019).

[31] M. B. Voloshin, Phys. Rev. D 100, 034020 (2019).

[32] S. Sakai, H.-J. Jing, and F.-K. Guo, Phys. Rev. D 100, 074007 (2019).

[33] Z. G. Wang and X. Wang, arXiv:1907.04582.

[34] Y. Yamaguchi, H. García-Tecocoatzi, A. Giachino, A. Hosaka, E. Santopinto, S. Takeuchi, and M. Takizawa, arXiv:1907.04684.

[35] M. Z. Liu, T. W. Wu, M. Sánchez Sánchez, M. P. Valderrama, L. S. Geng, and J. J. Xie, arXiv:1907.06093.

[36] Y.H. Lin and B.S. Zou, Phys. Rev. D 100, 056005 (2019).

[37] B. Wang, L. Meng, and S. L. Zhu, J. High Energy Phys. 11 (2019) 108.

[38] T. Gutsche and V.E. Lyubovitskij, Phys. Rev. D 100, 094031 (2019).

[39] T. J. Burns and E. S. Swanson, Phys. Rev. D 100, 114033 (2019).

[40] A. Ali and A. Y. Parkhomenko, Phys. Lett. B 793, 365 (2019).

[41] R. Zhu, X. Liu, H. Huang, and C. F. Qiao, Phys. Lett. B 797, 134869 (2019).

[42] Z. G. Wang, arXiv:1905.02892.

[43] J. F. Giron, R. F. Lebed, and C. T. Peterson, J. High Energy Phys. 05 (2019) 061.

[44] J. B. Cheng and Y. R. Liu, Phys. Rev. D 100, 054002 (2019).

[45] F. Stancu, Eur. Phys. J. C 79, 957 (2019).

[46] M. I. Eides, V. Y. Petrov, and M. V. Polyakov, arXiv:1904 .11616.

[47] C. Fernández-Ramírez, A. Pilloni, M. Albaladejo, A. Jackura, V. Mathieu, M. Mikhasenko, J. A. Silva-Castro, and A. P. Szczepaniak (JPAC Collaboration), Phys. Rev. Lett. 123, 092001 (2019).

[48] C. Hanhart, Y. S. Kalashnikova, P. Matuschek, R. V. Mizuk, A. V. Nefediev, and Q. Wang, Phys. Rev. Lett. 115, 202001 (2015).

[49] F.-K. Guo, C. Hanhart, Y. S. Kalashnikova, P. Matuschek, R. V. Mizuk, A. V. Nefediev, Q. Wang, and J.-L. Wynen, Phys. Rev. D 93, 074031 (2016).

[50] Q. Wang, V. Baru, A. A. Filin, C. Hanhart, A. V. Nefediev, and J.-L. Wynen, Phys. Rev. D 98, 074023 (2018). 
[51] V. Baru, E. Epelbaum, A. A. Filin, C. Hanhart, A. V. Nefediev, and Q. Wang, Phys. Rev. D 99, 094013 (2019).

[52] C. W. Xiao, J. Nieves, and E. Oset, Phys. Rev. D 88, 056012 (2013).

[53] M. Z. Liu, F.Z. Peng, M. Sánchez Sánchez, and M. P. Valderrama, Phys. Rev. D 98, 114030 (2018).

[54] M. B. Wise, Phys. Rev. D 45, R2188 (1992).

[55] T. M. Yan, H. Y. Cheng, C. Y. Cheung, G. L. Lin, Y. C. Lin, and H. L. Yu, Phys. Rev. D 46, 1148 (1992); 55, 5851(E) (1997).

[56] J. Hu and T. Mehen, Phys. Rev. D 73, 054003 (2006).

[57] M. Tanabashi et al. (Particle Data Group), Phys. Rev. D 98 , 030001 (2018) and the 2019 update.

[58] W. Detmold, C. J. D. Lin, and S. Meinel, Phys. Rev. D 85, 114508 (2012).

[59] V. Baru, E. Epelbaum, A. A. Filin, C. Hanhart, and A. V. Nefediev, J. High Energy Phys. 06 (2017) 158.

[60] U. Skerbis and S. Prelovsek, Phys. Rev. D 99, 094505 (2019).

[61] M. Albaladejo, F.-K. Guo, C. Hidalgo-Duque, and J. Nieves, Phys. Lett. B 755, 337 (2016).

[62] J.-J. Wu, T.-S. H. Lee, and B.-S. Zou, Phys. Rev. C 85, 044002 (2012).

[63] Y.-H. Lin, C.-W. Shen, F.-K. Guo, and B.-S. Zou, Phys. Rev. D 95, 114017 (2017).

[64] See the Supplemental Material at http://link.aps.org/ supplemental/10.1103/PhysRevLett.124.072001 for further details on the calculations and additional fits to check the stability of our results.
[65] T. J. Burns, Eur. Phys. J. A 51, 152 (2015).

[66] M. Pavon Valderrama, Phys. Rev. D 100, 094028 (2019).

[67] F.-K. Guo, U.-G. Meißner, W. Wang, and Z. Yang, Phys. Rev. D 92, 071502(R) (2015).

[68] X. H. Liu, Q. Wang, and Q. Zhao, Phys. Lett. B 757, 231 (2016).

[69] M. Bayar, F. Aceti, F.-K. Guo, and E. Oset, Phys. Rev. D 94, 074039 (2016).

[70] A. Ali et al. (GlueX Collaboration), Phys. Rev. Lett. 123, 072001 (2019).

[71] Q. Wang, X. H. Liu, and Q. Zhao, Phys. Rev. D 92, 034022 (2015).

[72] V. Kubarovsky and M. B. Voloshin, Phys. Rev. D 92, 031502(R) (2015).

[73] M. Karliner and J. L. Rosner, Phys. Lett. B 752, 329 (2016).

[74] A. N. Hiller Blin, C. Fernández-Ramírez, A. Jackura, V. Mathieu, V. I. Mokeev, A. Pilloni, and A. P. Szczepaniak, Phys. Rev. D 94, 034002 (2016).

[75] X. Cao and J. P. Dai, Phys. Rev. D 100, 054033 (2019).

[76] X. Y. Wang, X. R. Chen, and J. He, Phys. Rev. D 99, 114007 (2019).

[77] D. Winney, C. Fanelli, A. Pilloni, A. N. H. Blin, C. Fernandez-Ramirez, M. Albaladejo, V. Mathieu, V. I. Mokeev, and A. P. Szczepaniak (JPAC Collaboration), Phys. Rev. D 100, 034019 (2019).

[78] G. Rossi and G. Veneziano, arXiv:1909.01753. 